ORNL /TM-2007/25

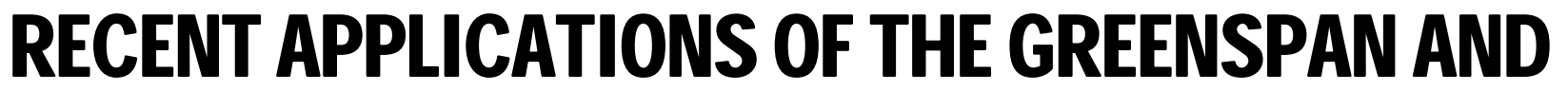

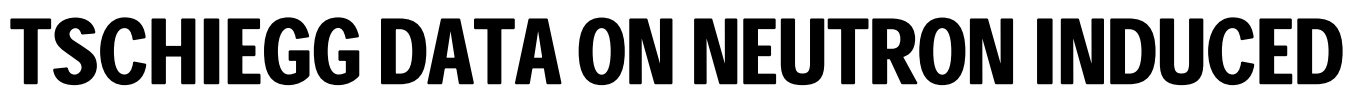
of

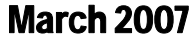


RECENT APPLICATIONS OF THE GRENSPAN AND TSCHIEGG DATA ON NEUTRON INDUCED CAVITATION THRESHOLDS

\author{
C.D.West
}

March 2007 
TABLE OF CONTENTS

Page

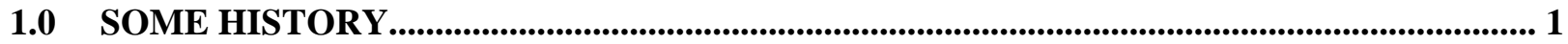

2.0 THE RADIATION INDUCED CAVITATION THRESHOLD PROBLEM ............................ 1

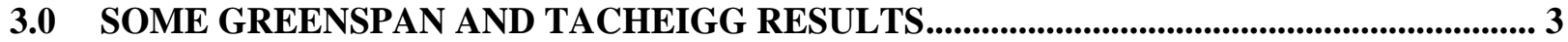

4.0 HAZARDS ASSESSMENT METHODOLOGY ........................................................................ 4

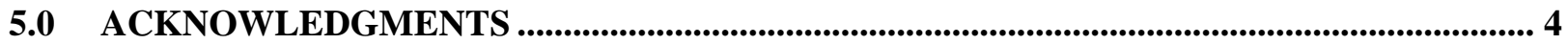

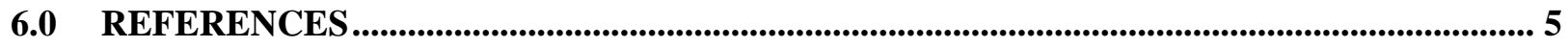




\section{RECENT APPLICATIONS OF THE GRENSPAN AND TSCHIEGG DATA ON NEUTRON INDUCED CAVITATION THRESHOLDS}

\subsection{SOME HISTORY}

In 1967 Greenspan and Tschiegg published a paper $^{1}$ on radiation induced acoustic cavitation. They researched the thresholds for cavitation induced in various liquids by fast neutrons, $\alpha$-decay recoils and fission fragments. It turns out that these data can be used to verify predictions of a more recent theory of radiation induced cavitation nucleation.

In 1979, in a report ${ }^{2}$ to their sponsor (The Office of Naval Research) they published new details of their results on neutron induced cavitation thresholds, including tables of the thresholds at different temperatures for various liquids. They were also some fission fragment results, but none of the $\alpha$-decay recoil data.

By that time Greenspan had evidently retired while I had left the field of cavitation research and did not know of the existence of their report [which also contains the only published record of some cavitation threshold measurements made by West and Howlett at Harwell, England].

Later still, in 1982, Greenspan and Tschiegg published the graphical data - but not the tables - in a more easily accessible form ${ }^{3}$.

In the late 1990s I revisited the problem (see below) of calculating radiation induced cavitation thresholds. There was interest in this because the Spallation Neutron Source (SNS) project, then just beginning, planned to use a liquid mercury target to produce intense bursts of neutrons when irradiated by a pulsed, high energy proton beam. It was known that the pressure waves produced by local heating when the proton pulse struck the target could, upon reflection at the walls of the mercury container, give rise to very high, although brief, negative pressure waves in the mercury. There was concern that cavitation might result and, if it did, might lead to undesirable effects ${ }^{4}$. With the encouragement of the SNS target team this author managed further to develop an earlier method of calculating the threshold for such cavitation $^{5}$, and the SNS project kindly provided funding to publish the work in two ORNL reports ${ }^{6,7}$. Around that period, I was also able to acquire a copy of Greenspan and Tschiegg's 1979 report $^{2}$ to which, fortunately, they had referred in their 1982 Acoustical Society of America note ${ }^{3}$.

Now retirement has given me opportunities to return to some work in the field of cavitation and to study some topics for my own interest - such as this one. And it is a pleasure to acknowledge that the Greenspan and Tschiegg publications still contain data, hitherto unused, that can bring new light to cavitation studies.

\subsection{THE RADIATION INDUCED CAVITATION THRESHOLD PROBLEM}

The first and most important application for bubble nucleation by energetic particles was the bubble chamber, invented in the 1950 s by Glaser, in which boiling is initiated in a superheated liquid. ${ }^{8}$ The liquid is, typically, at a temperature $1 / 2$ to $2 / 3$ of the way between its normal boiling point and its critical point. Boiling is prevented by applying an external pressure greater than the saturation pressure. When that external pressure is suddenly reduced, to below the saturation pressure, the liquid is superheated and wants to boil. Vapor bubbles form along the tracks of energetic particles passing through, or formed within, the chamber. 
By 1961, a thermal spike theory of such bubble nucleation was fully accepted, having been quantified by Seitz ${ }^{9}$, with the quantification verified experimentally, at least for the case of bubbles nucleated by the $\alpha$-decay of nuclei within the liquid ${ }^{10}$.

The Seitz theory can be thought of as having two parts. The first is the extremely general and, as far as we know, correct idea that the origin of the macroscopic bubbles is a localized "thermal spike" in the liquid, leading to a phase change. Even before Seitz' paper was published, this idea was gaining acceptance over the original concept that in the bubble chamber, as in the cloud chamber, the formation of the tracks depended upon electric changes separated by the incoming radiation.

Seitz went further, and quantified the thermal theory as applied to bubble chambers. An important part of his calculation was the idea of a "critical radius": if a vapor filled bubble or nucleation center of this critical size or greater could be created, then it would continue to grow, by evaporation, to a macroscopic size.

This critical radius is easily calculated. The effect of surface tension on the bubble is equivalent to an inwardly directed pressure $2 \sigma / r$, where $\sigma$ is the surface tension of the liquid against its vapor and $r$ the bubble radius. The bubble will shrink or grow according to whether the vapor pressure $\mathrm{P}_{\text {vap }}$ inside it is less or greater than the externally applied pressure $\mathrm{P}_{\mathrm{ext}}$ plus the surface tension effect. The critical radius is the one for which the two sets of forces are equal so

$$
\frac{2 \sigma}{r_{\text {crit }}}=\mathrm{P}_{\text {vap }}-\mathrm{P}_{\text {ext }}
$$

Knowing $r_{\text {crit }}$, one can calculate the critical bubble volume and therefore the energy needed to form it. The static energy terms, i.e. ignoring viscous losses and kinetic energy given to the liquid surrounding the bubble, are $(4 / 3) \pi r_{\text {crit }}^{3} \rho_{v} \mathrm{H}_{v}$, the enthalpy of evaporation (usually the largest term in bubble chamber-like conditions) and $4 \pi r_{c r i t} \sigma$, the surface energy. In these formulae, $\rho_{v}$ is the vapor density and $\mathrm{H}_{v}$ is the enthalpy of evaporation per unit mass. A discussion of these and the, usually smaller, dynamic terms is given in e.g. reference 6. This formation energy must be provided by the incoming energetic particle (in typical bubble chamber usage, the particles are very high speed and ionize the liquid atoms, producing delta rays that lose their energy in a small region around the particle track.)

In Riepe and Hahn's bubble chamber experiments ${ }^{10}$, the threshold pressure, $\mathrm{P}_{\text {ext }}$, needed to form bubbles with the (known) energy from alpha decay recoils was measured. These recoils are heavy and slow, with a well known energy that is lost in a track length that is usually less than the diameter of the critical bubble size, thus making it relatively simple to verify equation (1).

The formation energy calculated from equation (1) and the energy available from the $\propto$-decay agreed within a few percent. It was a triumph.

However, by the late 1950s it was also known that bubbles could be nucleated even in "cold" liquids, well below their normal boiling point, if they were subjected to a sufficiently negative pressure or tensile stress - for example, by intense acoustic excitation or by centrifugal force.

In 1961, Hahn reported the negative pressure thresholds, measured in a centrifugal apparatus, for bubble nucleation by $\alpha$-decay recoils ${ }^{11}$. The 1961 paper simply reported the results, but a later paper on acoustically excited cavitation nucleated by recoils from fast neutron collisions ${ }^{12}$ revealed a very great 
surprise. In one section, Hahn and Peacock discussed the cavitation mechanism and, as Riepe and Hahn had done for the bubble chamber, they compared the energy terms calculated from the critical radius model with the energy thought to be available from the recoils:

“. . . the energy loss of a carbon ion within two critical radii near the end of its path is approximately 100 $\mathrm{keV}$, which is six times larger than the sum of the $17 \mathrm{keV}$ for the static terms. A similar discrepancy for the $\alpha$-decay recoils was found at negative pressures in the centrifugal experiments."

The contrast with the stunningly close agreement between calculation and experiment in Riepe and Hahn's bubble chamber work could hardly have been greater. To the few physicists aware of it, this problem of the difference between the positive and negative pressure situations was a challenging puzzle.

A 1967 report $^{5}$ provided an explanation for the difference by recognizing that for liquids under a large negative pressure, the criterion of a critical radius - which had been derived as part of Seitz's study of positive pressure bubble chamber operation - was not a sufficient condition to ensure continuous growth from the initial small hot spot up to the critical size. The critical radius criterion led to growth from that radius on, but was not enough to ensure growth up to that point in cold, low vapor pressure liquids.

The analysis of bubble growth in reference 5 replaced the concept of a single critical radius with a requirement that the mass $\mathrm{m}$ and temperature $\mathrm{T}$ of the gas in the bubble be such that its pressure would be greater than the sum of the inwardly directed surface tension forces and the external pressure, at all stages of the growth (hence, it is referred to here as the $\mathrm{mT}$ theory). The calculations, which were carried out with many simplifications, predicted that in all positive pressure situations, and even in negative pressure cases where the magnitude of the negative pressure was less than twice the vapor pressure of the liquid, the Seitz critical radius calculation was applicable and appropriate. For greater negative pressures, a different, more stringent, set of conditions is required to ensure continued growth. All of the negative pressure data reported by Hahn in reference 11 fall into the latter category.

The purpose of these notes is to show that experimental measurements, made by Greenspan and Tschiegg, of the neutron induced cavitation threshold in Freon 11, fully support that prediction.

\subsection{SOME GREENSPAN AND TACHEIGG RESULTS}

Attachment 1, copied from reference 2, shows the $G \& T$ (Greenspan and Tschiegg) results for the cavitation threshold in Freon 11 irradiated by a Plutonium/Beryllium neutron source. There are eight results, covering the temperature range from $-50^{\circ} \mathrm{C}$ to $+50.5^{\circ} \mathrm{C}$. The normal boiling point of Freon 11, where the vapor pressure is of course approximately $1 \mathrm{bar}$, is $23.8^{\circ} \mathrm{C}$, and it turns out that in the five measurements at $18.6^{\circ} \mathrm{C}$ and below the magnitude of the cavitation threshold is more than twice the vapor pressure. For the three highest temperature readings, $\left|\mathrm{P}_{\text {threshold }}\right|$ is less than twice the vapor pressure - and in fact for the highest temperature point at $50.5^{\circ} \mathrm{C}$, the threshold is actually positive. Thus we have a set of consistent measurements, all made with the same apparatus and all but one made on the same day, that include points in the two different regimes described in the previous section.

The $G \& T$ tables include the standard deviations of their different measurements. In addition, they rounded their result to the nearest 0.1 bar in the table. For arithmetic simplicity, in this paper the standard deviation and the .05 bars maximum error from the rounding process are simply added to establish an approximate error bar when plotting their data (see fig. 1, below).

The Yaws data base ${ }^{13}$ was used to find the vapor pressure at the measurement temperatures (table 1 below) and at intermediate points for plotting. 
Table 1

\begin{tabular}{|c|c|c|c|c|c|}
\hline Temp ${ }^{\circ} \mathbf{C}$ & $\begin{array}{c}\text { Threshold } \\
\mathbf{P}_{\text {neg, bars }}\end{array}$ & $\begin{array}{c}\text { Errors } \pm \\
\text { bars }\end{array}$ & $\mathbf{P}_{\text {vap }}$ bars & $\left|\mathbf{P}_{\text {neg }}\right|$ bars & $\begin{array}{c}2 \text { P }_{\text {vap }} \\
\text { bars }\end{array}$ \\
\hline-50 & -5.8 & 0.40 & .0257 & 5.8 & .051 (mT Theory Applies) \\
\hline-31 & -5.8 & 0.16 & .0858 & 4.6 & 0.17 (mT Theory Applies) \\
\hline-10.9 & -3.6 & 0.20 & 0.2460 & 3.6 & 0.49 (mT Theory Applies) \\
\hline 3.8 & -3.0 & 0.10 & 0.4763 & 3.0 & 0.95 (mT Theory Applies) \\
\hline 18.6 & -2.5 & 0.15 & 0.8493 & 2.5 & 1.70 (mT Theory Applies) \\
\hline 32.1 & -1.4 & 0.10 & 1.3644 & 1.4 & 2.73 (Seitz Theory Applies) \\
\hline 41.5 & -0.4 & 0.06 & 1.8462 & 0.4 & 3.69 (Seitz Theory Applies) \\
\hline 50.5 & 0.9 & 0.12 & 2.4203 & $+\mathrm{ve}$ & 4.84 (Seitz Theory Applies) \\
\hline
\end{tabular}

Table 1 shows that for the three highest temperature measurements, the Seitz critical radius would be the appropriate way to calculate the expected threshold, because the negative pressure is less than twice the vapor pressure of the liquid, or the pressure is positive. In the other five cases the $\mathrm{mT}$ theory requires that a different criterion be used.

When these data are plotted (fig. 1, below) one sees that indeed the five lower temperature points lie on one smooth monotonic curve. The three highest temperature points lie on a different curve (the curves shown in the figure were plotted, by hand and by eye, using French curves.)

A similar picture can be obtained from the $\mathrm{G} \& \mathrm{~T}$ data for Freon 113 (figure 2, below), although because of the greater scatter, especially on the lower temperature data points, in the Freon 113 results the difference between the two regimes is not quite so stark. Because of the considerable scatter, rather than trying to draw a curve, by eye, through the lower temperature data points, the Tk Solver program was used to find a linear regression, least squares fit: the $\mathrm{R}^{2}$ correlation coefficient is 0.92 .

\subsection{DISCUSSION}

A 1967 report $^{5}$ explained qualitatively why Hahn's measured values of the negative pressure threshold for bubble formation by $\alpha$-decay recoils could disagree with predictions based on the critical radius criterion, even though the latter had been superbly successful in the bubble chamber case.

The new approach seems to be supported, in its most general prediction, by the experimental measurements of Greenspan and Tschiegg, as described above.

The 1967 theory $^{5}$, with all its simplifications, did not disagree as badly as the critical radius model when quantitative calculations of the bubble formation energy were compared with experimental results, but there was still an order of magnitude discrepancy. The 1998 ORNL $^{6}$ report greatly refined and extended the earlier calculations and brought much better - perhaps even satisfactory - agreement between theory and experiment; but it still came nowhere near the superb (within a few percent) agreement achieved in Ripe and Hahn's 1961 work on a (positive pressure) bubble chamber.

\subsection{ACKNOWLEDGEMENTS}

The author would like to thank Dr. Art Ruggles, Ellen Fisher, and Dr. William Bugg for their encouragement and for their considerable help in the preparation of this manuscript. 


\subsection{REFERENCE}

1. M. Greenspan and C.E. Tschiegg, "Radiation-Induced Acoustic Cavitation; Apparatus and Some Results," Jnl. Res. National Bureau of Standards - C. Engineering and Instrumentation, 71C, 4, 299 312 (1967).

2. M. Greenspan and C.E. Tschiegg, "Radiation-Induced Acoustic Cavitation; Threshold Versus Temperature for Some Liquids," Report NBSIR-79-1753 to ONR, 1 (1979).

3. M. Greenspan and C.E. Tschiegg, "Radiation-Induced Acoustic Cavitation; Threshold Versus Temperature for Some Liquids,” J. Acoust. Soc. Am., 72 (4), 1327-1331 (1982).

4. J.M. Carpenter, "Comments on the Possibility of Cavitation in Liquid Metal Targets for Pulsed Spallation Neutron Sources," International Workshop on the Technology and Thermal Hydraulics of Heavy Liquid Metals, Schruns, Austria, March 1996, OSTI ID 279677.

5. C. West, "Cavitation Nucleation by Energetic Particles," AERE-R5486, 1 (1967).

6. C.D. West, "Cavitation Bubble Nucleation by Energetic Particles," ORNL/TM-13683, 263 (1998).

7. C.D. West, "Cavitation in a Mercury Target," ORNL/TM-2000, 263 (2000).

8. D.A. Glaser, "The Bubble Chamber," Encyclopedia of Physics, Vol. XLV, 316-341, Nuclear Instrumentation II. S. Fluggeld, Springer-Verlag (1958).

9. F. Seitz, "On the Theory of the Bubble Chamber," Phys. Fluids, 1-1, 2-13 (1958).

10. G. Riepe and B. Hahn, "Untersuchungen zum Mechanismus der Blasenbildung in Freon-12 und Propan durch Ruckstosskerne einiger $\alpha$-Strahler," Helv. Phys. Acta, 34,8, 865-892 (1961).

11. B. Hahn, "The Fracture of Liquids Under Stress Due to Ionizing Particles," Nuovo Cimento, 2. 650653 (1961).

12. B. Hahn and R.N. Peacock, "Ultrasonic Cavitation Induced by Neutrons" Nuovo Cimento, 28, 2, 334340 (1963).

13. C.L. Yaws, "Floppy Disk Databases of Thermodynamic and Transport Properties" TREI, 658 Birchwood, Port Neches, Texas 77651 (1966). 
Attachment 1

\begin{tabular}{|c|c|c|c|c|c|c|c|c|}
\hline Date & Temp. & Frequency & $\begin{array}{l}\text { No of } \\
\text { data } \\
\end{array}$ & Range & R.S.D. ${ }^{\mathrm{C}}$ & $\begin{array}{l}\text { Thresho } \\
\text { (absolu } \\
\text { Value }\end{array}$ & $\frac{1 \mathrm{e})}{\text { S.D. }}$ & $\begin{array}{c}\text { Additional } \\
\text { Notes } \\
\end{array}$ \\
\hline 1969 & ${ }^{\circ} \mathrm{C}$. & $\mathrm{kHz}$ & & $10^{5} \mathrm{~Pa}$ & $10^{5} \mathrm{~Pa}$ & $10^{5} \mathrm{~Pa}$ & $\%$ & $f$ \\
\hline $7 / 2$ & -50 & 35.4 & 8 & $0-4.1$ & 0.14 & 5.8 & 6.1 & $8, \mathrm{~h}$ \\
\hline $6 / 24$ & $-3 !$ & 38.3 & 10 & $0-5.5$ & .07 & 4.6 & 2.3 & 8 \\
\hline $6 / 24$ & -10.9 & 36.5 & 10 & $0-5.5$ & .11 & 3.6 & 4.1 & g \\
\hline $6 / 24$ & 3.8 & 35.0 & 10 & $0-5.5$ & .04 & 3.0 & 1.5 & 8 \\
\hline $6 / 24$ & 18.6 & 33.7 & 10 & $0-5.5$ & .09 & 2.5 & 4.0 & $g$ \\
\hline $6 / 24$ & 32.1 & 25.0 & 10 & $0.7-3.4$ & .04 & 1.4 & 3.8 & 8 \\
\hline $6 / 24$ & 41.5 & 24.1 & 10 & $1.4-4.1$ & .02 & 0.4 & 3.4 & $\varepsilon$ \\
\hline $6 / 24$ & 50.5 & 23.3 & 10 & $2.1-4.8$ & .04 & -.9 & 7.9 & 8,1 \\
\hline
\end{tabular}

(a) to (f) See footnotes to Table 2.

(B) In $125 \mathrm{ml}$ conical flask driven by leahd zirconate-titanate cylinder, $1.5 \mathrm{~cm} \mathrm{D.}$ by $2.5 \mathrm{~cm} \mathrm{high.} \mathrm{See} \mathrm{F1g.} 3$ of [1].

(b) Cell not stable at higher overpressures.

(i) Cell not stable at lower overpressures.

Copied from: M. Greenspan and C.E. Tschiegg "Radiation-Induced Acoustic Cavitation Threshold vs. Temperature For Some Liquids,” Report NBSIR-79-1953 to ONR (NBS, May 1979) 
Figure 1.

Freon 11. G\&T threshhold data with CDW error

harc Smnoth ciorve is 2 y vannr nroccioro 